\title{
AGC GROUP
}



\section{CAMK GROUP}

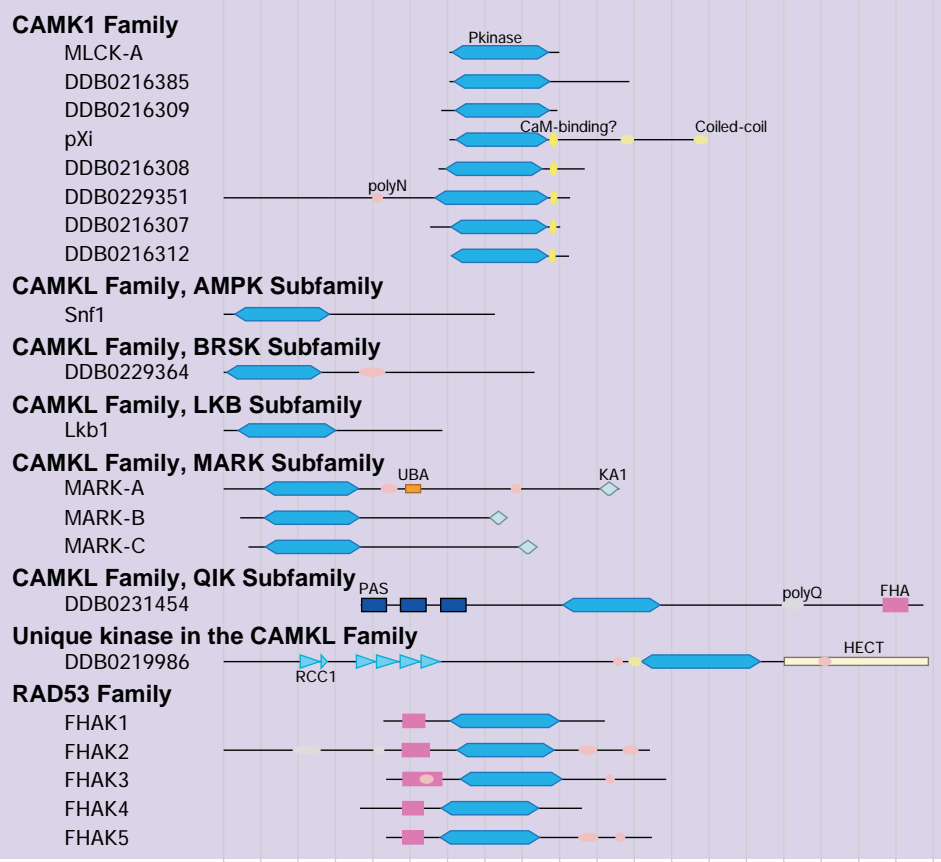

\section{CK1 GROUP}

\section{CK1 Family \\ CK1, C2dup \\ TTBK Family \\ DDB0216336}

\section{CMGC GROUP}

\section{CDK Family, CDC2 Subfamily $\mathrm{Cdc} 2$ \\ CDK Family, CDK5 Subfamily Cdk5 \\ CDK Family, CDK7 Subfamily Cdk7 \\ CDK Family, CDK8 Subfamily Cdk8}




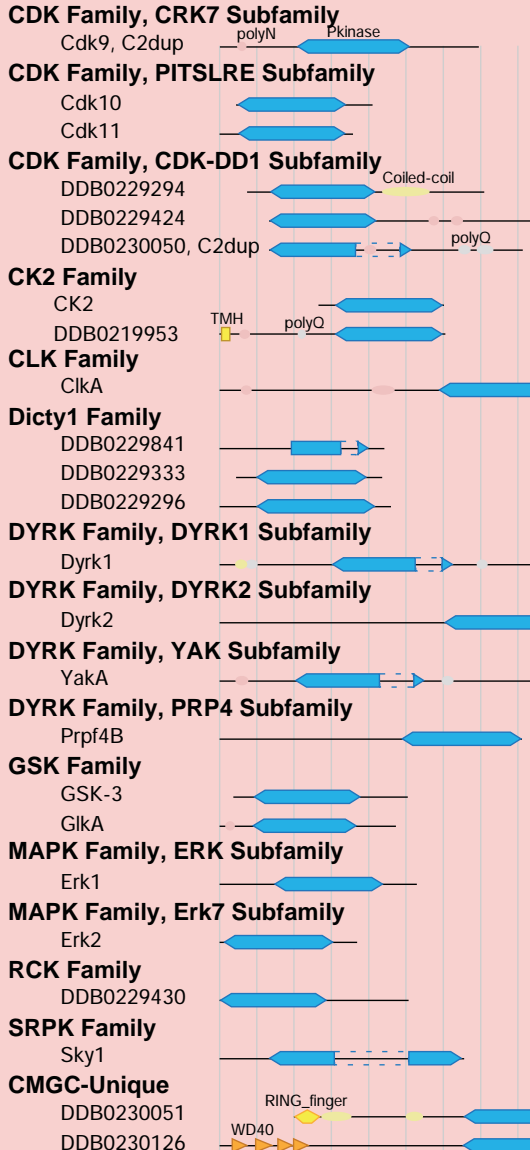

ramily

\section{STE GROUP}

Dicty2 Family

DDB0229896

DDB0229971

DDB0231212

DDB0229915

DDB0229877

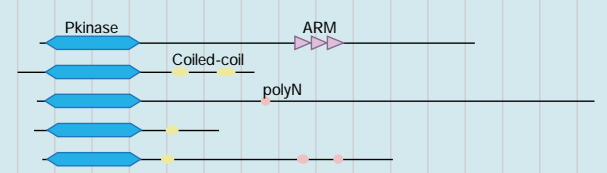

Dicty3 Family, FNIPK Subfamily

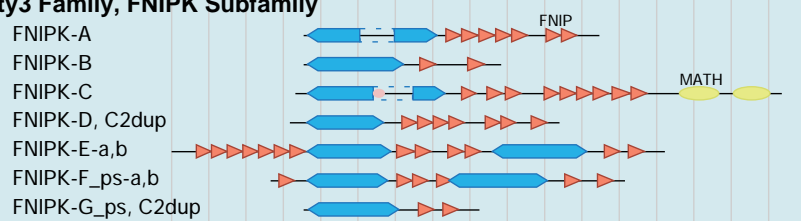

FNIPK-G ps, C2dup

Dicty3 Family, NZAK Subfamily
DPYK4-a
r
(C-terminal domain is classified as TKL/CZAK)
ZakA-a
(C-terminal domain is classified as TKL/CZAK)

Unique kinases in the Dicty3 Family

DDB0230002

DDB0229378

STE7 Family

Mek1

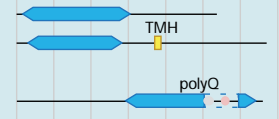

STE11 Family

MEKKalpha

TE20 Family, FRAY Subfamily

DDB0230012

DDB0229911

STE20 Family, MKC Subfamily

MkcA

$\mathrm{MkCB}$

$\mathrm{MkcC}$

$\mathrm{MkCD}$

$\mathrm{MkcE}$

$\mathrm{MkCF}$

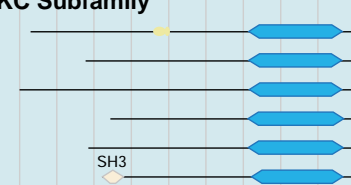

STE20 Family, PAKA Subfamily

PakA

PakB

PakC

PakD

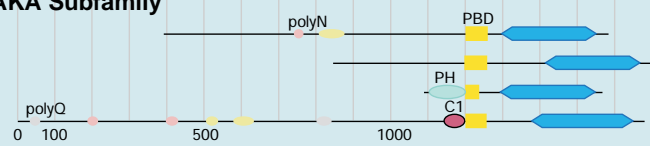




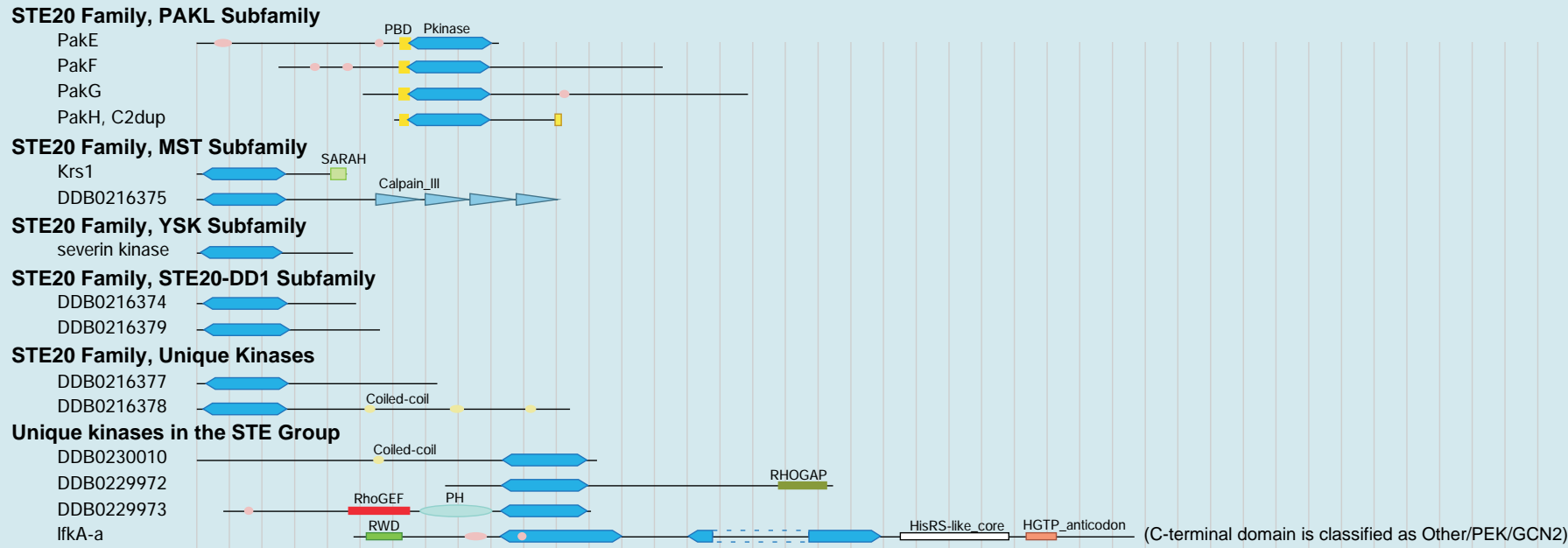

\section{TKL GROUP}

ARMK Family

DDB0230038 DDB0230124

ARK Family

ARCK-1

SAPKalpha, C2dup

DDB0229849

DDB0229848

Phg2

DDB0229847

- Coiled-coil

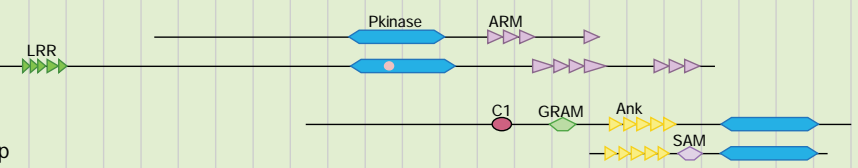

DDB0229871-b

DDB0229844

DDB0229850

CZAK Family

DPYK4-b (N-terminal domain is classified as STE/Dicty3/NZAK)

ZakA-b
SpIA

DDB0229872

7TMK1

Dicty4 Family, DRK Subfamily

rk1

rk2

rk3

DDB0214883
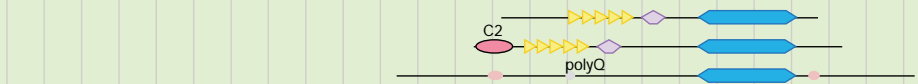

RGS

( $\mathrm{N}$-terminal domain is classified as TKL/Unique)

Dicty4 Family, SHK Subfamily

Shk1

Shk2

Shk3

Shk4

Shk5

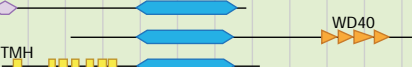

DDB0229963

DDB0231199

Dicty5 Family

DDB0229940

DDB0218878e

Rck1

DDB0231197

DPYK Family

DPYK2

DPYK3-b

Gdt Family

Gdt1

Gdt2

Gdt3_ps

Gdt4

Gdt6

Gdt8

Gdt9

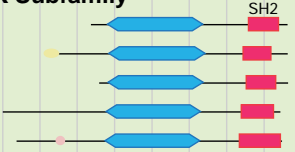

signal_peptide drk_ex TMH


LISK Family, LISK-DD1 Subfamily
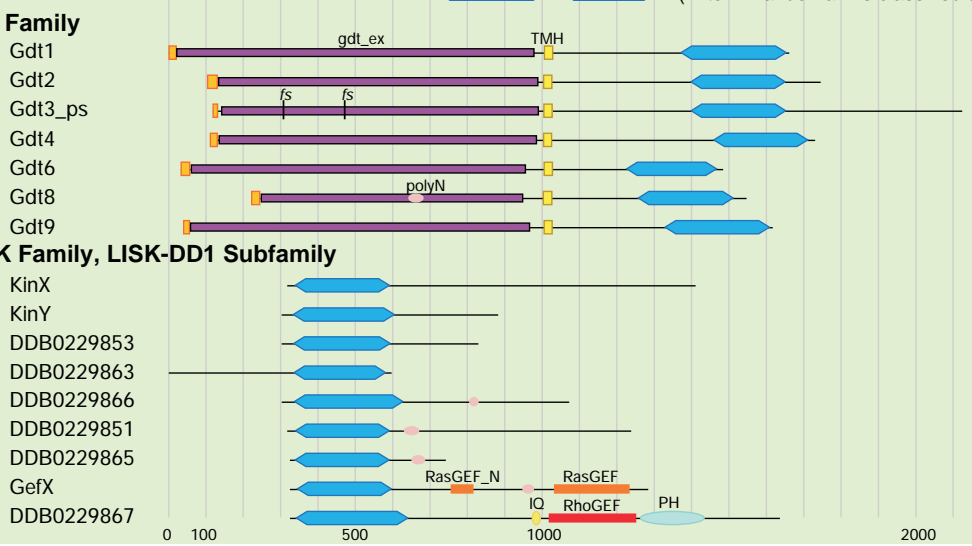

1000 
MLK Family, HH498 Subfamily DDB0230019 ROCO Family

GbpC

pats1

qkgA, C2dup

Roco4

Roco5

Roco6

Roco7

Roco8

Roco9

Roco10

Roco11
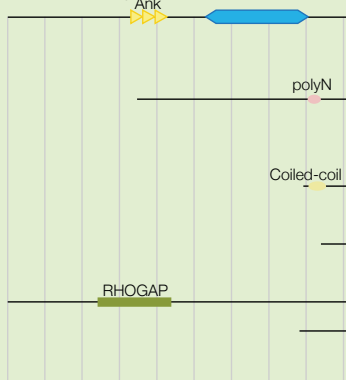

inases in the TKL Group

DPYK3-a

DDB0229956

DDB0229957

DDB0220138

DDB0220436

DDB0230133

DDB0229871-a

DDB0229955

DDB0230119ps

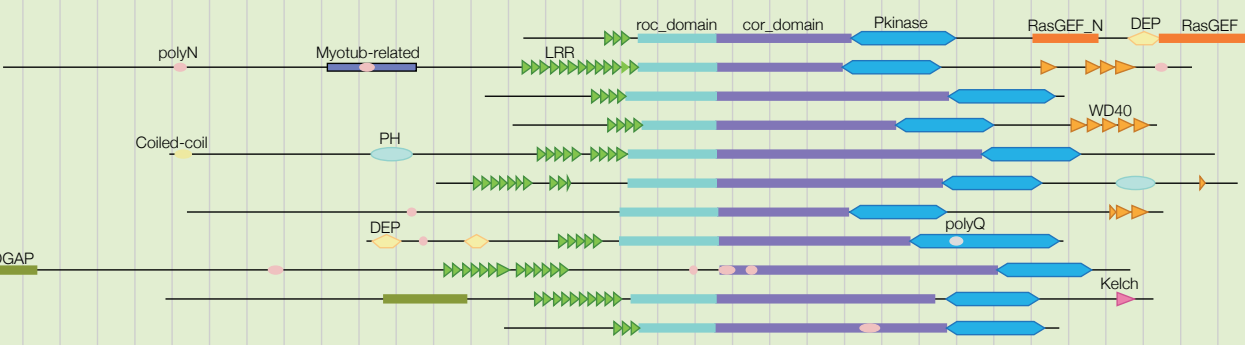

\section{OTHER GROUP}

\section{AUR Family \\ Aurora \\ BUB Family \\ Bub1 \\ BUD32 Family \\ Bud32}

CAMKK Family, Meta Subfamily

DDB0220010

CDC7 Family

Cdc7

Dicty6 Family

DDB0231196

DDB0229339

DDB0204413ps

Dicty7 Family

DDB0231195

DDB0229335

Dicty8 Family

DDB0216331

DDB0229344

Dicty9 Family

DDB0231281-a,b-

DDB0219988-a,b-

DDB0230037-a,b- - -

Dicty10 Family

DDB0204911e

DDB0230125

DDB0231304ps

DDB0204909ps

DDB0187322ps

Haspin Family

DDB0231182

IKS Family

IksA

IRE Family

IreA

IrlA

IrlB, C2dup

IrIC

IrID

$|r| E$

IrlF, C2dup

NAK Family

DDB0229350

DDB0216373

DDB0229347

NEK Family

Nek2

Nek3

Nek4

DDB0229345

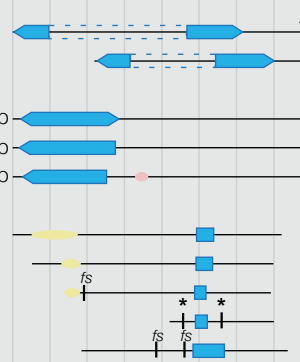

PEK Family, PEK Subfamily

DDB0220611e

DDB0229432
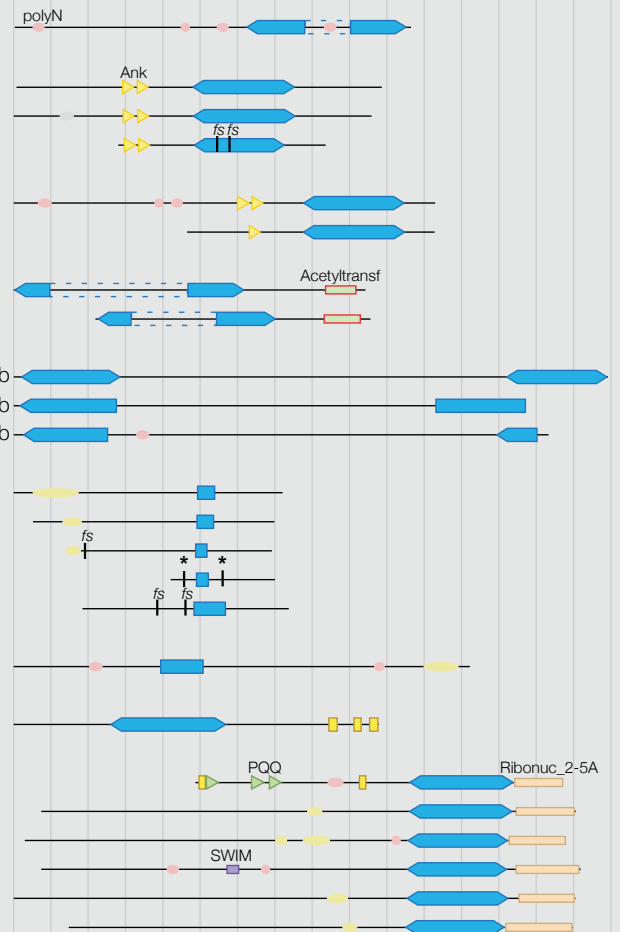

Unique kinase in the PEK Family DDB0220652

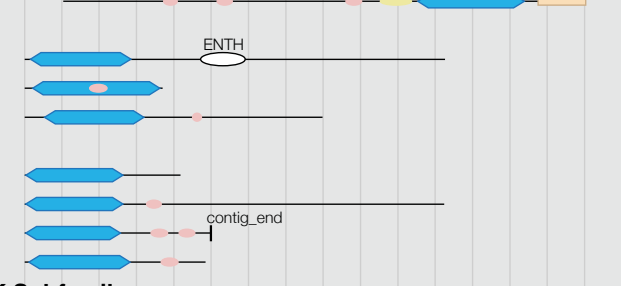


PEK Family, GCN2 Subfamily

IfkA-b

IfkB

DDB0216407

PLK Family

PLK

SAMK Family

SAMK-A

SAMK-B

samkB_ps

SAMK-C

SAMK-D

samkE_ps1

samkE_ps2

SCY1 Family

Scy1

Scy2

SLOB Family

Slob1

Slob2

TBCK Family

TBCK

TTK Family

TtkA

ULK Family

Apg1

Tsunami

VPS15 Family Vps15

WEE Family

DDB0220004

DDB0216398

DDB0229384

Unique kinases

DDB0229381

DDB0231198

7TMK2

DDB0229334

DDB0231326

DDB0231179

DhkG

DDB0230007

DDB0229346

LVSG

DDB0231335

DDB0231559

DDB0216536ps

DDB0229337

DDB0204419ps

DDB0187382ps



\section{ATYPICAL GROUP}

ABC1 Family, ABC1-A Subfamily

AdckA

ABC1 Family, ABC1-B Subfamily

AdckB1

AdckB2

ABC1 Family, ABC1-C Subfamily

AdckC

AFK Family

DDB0231760

DDB0233254

DDB0233255

DDB0233252

Alpha Kinase Family, MHCK Subfamily

MHCK-A

MHCK-B

MHCK-C

MHCK-D

Alpha Kinase Family, Unique Kinases

ak1

VwkA

BRD Family

DDB0220693

DDB0220694

G11 Family

G11

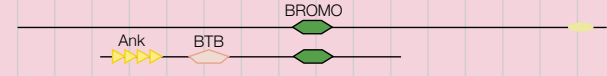

Coiled-coil Alpha

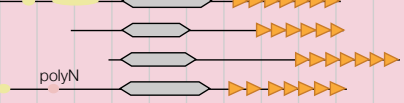

poly

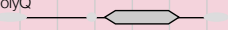

wwa CaM-binding

$\begin{array}{lll}0 & 100 & 500\end{array}$

1000

2000

3000

4000 
PIKK Family, DNAPK Subfamily DNAPKCS

PIKK Family, FRAP Subfamily

\section{tor}

PIKK Family, SMG1 Subfamily SMG1

PIKK Family, TRRAP Subfamily TRRAP

polyN

polyQ

PIKK Family, ATR Subfamily

ATR

RIO Family, RIO1 Subfamily Rio1

RIO Family, RIO2 Subfamily

Rio2

TAF1 Family

TAF1

$+\square_{-}$

Family

$\longrightarrow$

- BROMO

Coiled-coil

DDB0220714 RiNG_finger BBOX Kelch

\section{HISTIDINE KINASES}

DhkA

DhkB

DhkC

DhkD

DhkE

DhkF

DhkG

DhkH

Dhkl, C2dup

DhkJ

DhkK

DhkL

DhkM

DokA

500

1000

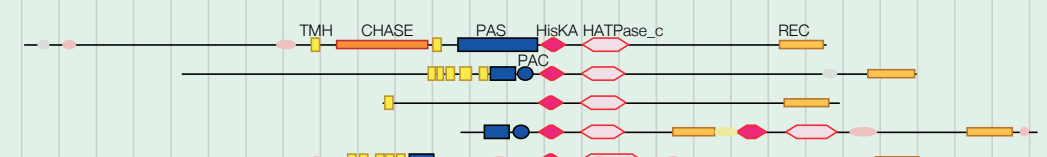

Pkinase

AAA
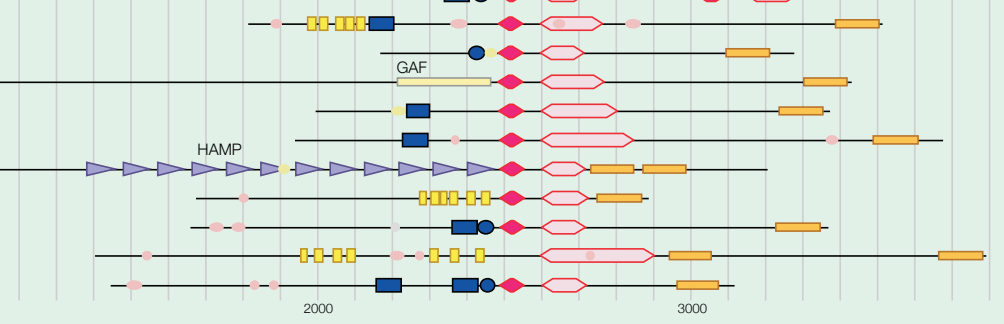
polyN Regions at least 19 aa in length that are at least $90 \%$ asparagine.

polyQ Regions at least 19 aa in length that are at least $90 \%$ glutamine.

Coiled-coil Regions predicted to dimerize by forming parallel alpha-helices.

signal_peptide

$$
\text { TMH Transmembrane helix. }
$$

\section{KINASE CATALYTIC DOMAINS}

Pkinase Eukaryotic protein kinase (ePK) catalytic domain. Members phosphorylate

serine/threonine and/or tyrosine.

kinase_C Domain found downstream of many ePK domains from the AGC Group.

ADF Actin Depolymerization Factor. Severs actin filaments and binds to actin monomers.

ABC Named for $A B C 1$ from yeast, and is unrelated to the $A B C$ transporter proteins.

$\mathrm{PI} 3 \mathrm{KC}$ Phosphoinositide 3-kinase isoforms include lipid kinases and protein kinases.

Alpha kinase A novel protein kinase catalytic domain with a related structure, but no sequence homology, to ePKs.

$\mathrm{RIO}$ Domain found in eukaryotes and prokaryotes that is related to ePKs.

BROMO May be involved in protein-protein interactions in transcriptional activation complexes.

HATPase_C Domain found in several ATP-binding proteins, including histidine kinases.

HisKA Dimerisation and phosphoacceptor domain of histidine kinases.

\section{EXTRACELLULAR DOMAINS} Domain found in the extracellular portion of receptor-like proteins and is
pHASE

gdt_ex Extracellular domain found in Dictyostelium GDT proteins.

drk_ex Extracellular domain found in two Dictyostelium protein kinases.

TIG Domain found in cell surface receptors such as Met and Ron, as well as in intracellular transcription factors, where it is involved in DNA binding.

\section{DOMAINS INVOLVED IN G-PROTEIN SIGNALING}

ARFGAP Putative zinc fingers with GTPase activating proteins (GAPs) towards the small

RHOGAP GTPase activator proteins towards Rho/Rac/Cdc42-like small GTPases.

TBC Thought to be a GTPase activator of Rab-like small GTPases.

RasGEF_N Found N-terminal to the RasGEF domain in several exchange factors for Ras-like RasGEF_N small GTPase.

RasGEF Guanine nucleotide exchange factor for Ras-like small GTPases.

RhoGEF Guanine nucleotide exchange factor for Rho/Rac/Cdc42-like GTPases.

RGS Regulator of G Protein Signalling. Promotes GTP hydrolysis by the alpha

RGS subunit of heterotrimeric G proteins.

roc_domain Ras-like domain of complex proteins.

cor_domain Domain found C-terminal of roc_domain.

PBD Domain that binds Cdc42p- and/or Rho-like small GTPases.

\section{SECOND-MESSENGER-BINDING DOMAINS}

CaM-binding? Putative $\mathrm{Ca}^{2+} /$ calmodulin binding domain.

IQ $\mathrm{Ca}^{2+-i n d e p e n d e n t ~ c a l m o d u l i n ~ b i n d i n g ~ d o m a i n . ~}$

cNMP_binding cAMP or cGMP binding site.

$\mathrm{PH} \quad$ Pleckstrin Homology. Binds inositol phosphates, and various proteins.

PX Phox domain. Phosphoinositide-binding modules with varying lipid-binding specificities.

FYV Fab1, YOTB/ZK632.12, Vac1, and EEA1. Zn²+-binding; binds Ptdlns(3)P. C1 Zn2+-binding domain that may bind to molecules such as diacylglycerol and phorbol esters.

$\mathrm{Ca}^{2+-}$-binding motifs that appears to bind phospholipids, inositol polyphosphates, and intracellular proteins.

\section{REPEATED DOMAINS}

Ank Ankyrin repeats are usually involved in protein-protein interactions.

ARM Armadillo repeats mediate protein-protein interactions.

$\mathrm{PQQ}$

Calpain_III

Domain found in several enzymes which utilise pyrrolo-quinoline quinone as a prosthetic group. Function is unknown.

The function of domain III of calpains is unknown.

WD40 Named for their 40 aa length, and because they often terminate in a WD

$\gg \rightarrow \quad$ dipeptide. Mediate protein-protein interactions, including to G $\beta$ and myosin II.

FNIP

$\gg \rightarrow$

$\rightarrow$ HAMP



LIM

LRR

MORN

MORN

RCC1
Named after the pattern of conserved residues. Found only Dictyostelium. Named for four of the proteins it is found in: histidine kinases, adenylyl cyclases, methyl binding proteins and phosphatases.

Named after the protein in which it was first identified. Its function is unknown.

Named after Lin-11 Isl-1 Mec-3. Binds two Zn²+; mediates protein-protein interactions.

Leucine-rich repeats provide a versatile structural framework for the formation of protein-protein interactions.

"Membrane Occupation and Recognition Nexus". Function is unknown. Repeated domain found in regulator of chromosome condensation (RCC). Binds to chromatin and acts as a guanine-nucleotide dissociation stimulator for Ran.

\section{OTHER CATALYTIC DOMAINS}

AAA ATPase family associated

Acetyltransf Gcn2-related N-actyltransferase. A superfamily that includes histone acetylases HECT

tRNA-synt_2b tRNA synthetase class II core domain. tRNA synthetases catalyse the

Myotub-related Domain in myotubularin-related proteins. Myotubularin is a lipid phosphatase

DSPC Dual-specificity (Ser/Thr and Tyr) protein phosphatases.

Ribonuc_2-5A An endoribonuclease that cleaves an intron from Hac1 mRNA in humans, which causing it to be more efficiently translated.

\section{PHOSPHOAMINO ACID-BINDING DOMAINS}

SH2 Src homology 2. Binds phosphotyrosine-containing polypeptides

FHA Forkhead-associated. Binds phosphopeptides. Highest specificity for phosphoFHA threonine, but also recognises phosphotyrosine.

\section{DOMAINS MEDIATING PROTEIN-PROTEIN INTERACTIONS}

BTB BR-C, ttk and bab. Mediates homomeric, and in some instances heteromeric,

BTB dimerization.

FBOX A motif found in cyclin-F. Serves as a link between a target protein and a ubiquitin-conjugating enzyme.

Filamin repeat These form a rod-like structure in filamin, which is an actin-binding protein.

$\mathrm{SH} 3$ Src homology 3. Binds to target proteins through sequences containing proline

PB1 Phox and Bem1p domain. This is present in many eukaryotic cytoplasmic

signalling proteins. Forms heterodimers.

mob_binding Binding site for Mob1. NDR Family kinases are activated by Mob1binding

Rhodanese Rhodanese is a sulfuryltransferase with two Rhodanese domains. Inactive

versions are found in dual specificity phosphatases and ubiquitin hydrolases.

RING_finger A specialised type of $Z n$-finger. It probably mediates protein-protein interactions

and probably has E3 ubiquitin-protein ligase activity.

DEP dishevelled, Egl-10, and pleckstrinproteins. Unknown function.

SAM Sterile Alpha Motif. Form homo- and hetero-oligomers. Also bind to non-SAM

SAM domain-containing proteins with a low affinity constant, and appear to bind RNA

SARAH Sav/Rassf/Hpo - 3 classes of eukaryotic tumour suppressors that it is found in.

Mediates homodimerization.

WH2 Wiskott Aldrich syndrome homology region 2. Mediates actin-binding.

POLO BOX Found in Polo kinases. Mediates interaction with multiple proteins, some of which are substrates.

\section{OTHER FUNCTIONS} HGTP_anticodon Found in Histidyl, Glycyl, Threonyl and Prolyl tRNA synthetases. It is probably

PAC Occurs C-terminal to a subset of PAS motifs, and is proposed to contribute to

PAS Found in Per, Arnt, Sim. Mediate protein-protein interactions, dimerization, and REC Receiver domain. Contains a phosphoacceptor site that is phosphorylated by

\begin{tabular}{|c|c|}
\hline \multicolumn{2}{|c|}{ UNKNOWN FUNCTION } \\
\hline BBOX & $\begin{array}{l}\text { Zn finger. Found in transcription factors, ribonucleoproteins and proto- } \\
\text { oncoproteins, but no function is clearly assigned to this domain. }\end{array}$ \\
\hline SWIM & Zn-finger. \\
\hline Beach & $\begin{array}{l}\text { Found in the BEIGE and CHS protein. The function is unknown. Usually } \\
\text { followed by WD repeats. }\end{array}$ \\
\hline RWD & Found in proteins containing Ring finger and WD40 repeat domains. \\
\hline Extensin_2 & $\begin{array}{l}\text { Homologous hydroxyproline-rich glycoproteins (HRGPs) found in the plant } \\
\text { extracellular matrix. }\end{array}$ \\
\hline$\stackrel{\text { ENTH }}{\complement}$ & $\begin{array}{l}\text { Epsin N-terminal homology. Found in proteins involved in endocytosis and } \\
\text { cytoskeletal machinery. May bind Ptdlns }(4,5) \mathrm{P} 2 \text { and Ptdlns }(1,4,5) \mathrm{P} 3 \text {. }\end{array}$ \\
\hline FAT & FRAP, ATM and TRRAP. Present in the PIK-related protein kinases. \\
\hline FAT-C & Found at the C-terminal end of the PIK-related protein kinases. \\
\hline GAF & $\begin{array}{l}\text { Found in phytochromes and cGMP phosphodiesterases (PDEs). In PDEs, } \\
\text { it forms an allosteric cGMP binding site. }\end{array}$ \\
\hline KA1 & Found in the C-terminal extremity of kinases in the MARK subfamily. \\
\hline GRAM & $\begin{array}{l}\text { Found in glucosyltransferases, myotubularins and other putative membrane- } \\
\text { associated proteins. }\end{array}$ \\
\hline HEAT_REPEAT & $\begin{array}{l}\text { Found in Huntingtin, EF3, PP2A regulatory subunit, and yeast TOR1. Related to } \\
\text { armadillo repeats. }\end{array}$ \\
\hline MATH & $\begin{array}{l}\text { Meprin And TRAF-Homology. TRAFs are intracellular proteins, and meprins are } \\
\text { extracellular. }\end{array}$ \\
\hline UBA & $\begin{array}{l}\text { Ubiquitin associated domain. Found in several proteins having connections to } \\
\text { the ubiquitination pathway. }\end{array}$ \\
\hline SPRY & Found in SplA and Ryanodine Receptor. \\
\hline vwa & $\begin{array}{l}\text { von Willebrand factor type A. WWA domains in extracellular eukaryotic proteins } \\
\text { mediate adhesion. Their function in intracelllular proteins is unknown. }\end{array}$ \\
\hline
\end{tabular}

